\title{
A Percolative Approach to Transport and Excess Noise in Polyacene Semiconductors
}

\author{
C. Pennetta, A. Carbone *, M. Tizzoni * and L. Reggiani \\ Dipartimento di Ingegneria dell'Innovazione, Università del Salento and CNISM, Via Arnesano, 73100, Italy \\ * Dipartimento di Fisica, Politecnico di Torino and CNISM, 10129 Torino, Italy \\ e-mail: cecilia.pennetta@unisalento.it
}

\begin{abstract}
Electrical transport measurements performed in polyacene thin films as a function of the applied voltage, pointed out the existence of a sharp peak in the relative spectral density of current noise occurring at voltages corresponding to the region of crossover between the Ohmic regime and the space charge limited current regime. Recently we have proposed an explanation of these experimental findings in terms of noise due to trapping and detrapping processes of the injected carriers by deep traps. This interpretation was carried out by adopting a phenomenological model making use of the measured current-voltage characteristics. To check this interpretation we have developed a new percolation model which will be discussed here.
\end{abstract}

\section{INTRODUCTION}

The injection of charge carriers at the moleculemetal interfaces and their trapping-detrapping (TD) processes at defect states crucially control the performances of organic semiconductor devices [1], [2]. Many studies in the literature investigated the effect of thermal and electrical stresses on TD processes in organic materials [3], [4], [5], [6], [7], [8], [9], [10], [11], [12], [13]. The largest part of these works focused on conduction properties and only a relatively small number was devoted to the study of the electrical noise [14], [15], [16].

Recent transport measurements in polyacene organic semiconductors [14] have evidenced a strong superlinear increase of the current voltage (I-V) characteristic curve and a concomitant peak of the relative spectral density of the low frequency current noise at voltages corresponding to the region of crossover between Ohmic and space charge limited currents (SCLC) regimes [14], [17]. An interpretation of these experiments has been proposed in Ref.
[14] in terms of a continuous percolation between two asymptotic regimes (Ohmic and SCLC), considered as different conducting phases. The noise increase was then explained as due to the clustering of insulating regions among which current paths are constrained [14]. Very recently, we have proposed a complementary interpretation of these experimental findings, in terms of noise due to trapping and detrapping (TD) processes of injected carriers by deep traps in the trap filling transition (TFT) regime [18].

This interpretation was based on a phenomenological model which makes use of the measured $I-V$ characteristic to estimate the fraction of filled traps as a function of the applied voltage. Then, the value of this fraction is used to express the spectral density of current noise in the TFT regime. The good agreement between the behaviors obtained from the phenomenological model and the experiments supports the idea of abscribing the sharp peak of noise in the TFT region to the fluctuating occupancy of the traps due to TD processes [18]. To further check this interpretation we have developed a new percolation model that is discussed together with its results in the next section.

\section{MODEL}

The model is based on the well known resistor network (RN) approach [19], [20] and it describes the semiconducting film as a binary and twodimensional resistor network. Precisely, here we consider a square-lattice with square geometry (size $N \times N)$. Each elementary resistor $r_{i, j}$ describes a small region of the film with homogeneous electrical properties, i.e. a small region characterized by a leading mechanism of electrical transport. This 
leading mechanism of transport defines the "state" of the resistor.

In the present model we assume that a resistor can be found in two states: an Ohmic state (state 1) with resistance $r_{1}$ and a SCLC state (state 2) with resistance $r_{2}$. We indicate with $N_{1}$ and $N_{2}$ the number of resistors in the states 1 and 2, with $N_{\text {tot }}=N_{1}+N_{2}=2 N^{2}$ the total number of resistors in the network. The fraction of resistors in the state $2, p=N_{2} / N_{\text {tot }}$, is the key quantity which characterizes the global network state: for $p>p_{c}$ the SCLC phase associated with the $r_{2}$ resistors percolates through the network, where $p_{c}$ is the percolation threshold [19].

An external constant voltage $V$ is applied to the RN through perfectly conducting bars placed at the left and right hand sides. As a consequence of this external bias, a voltage drop $v_{i, j}$ is also applied to each elementary resistor through which flows a current $i_{i, j}$, while $I$ is the total current flowing in the network. The network is taken to be in contact with a thermal bath at temperature $T$. Furthermore, to mimic the effect of trapping and detrapping processs, we assume the existence of random transitions between the two resistor states, occurring with probabilities:

$$
W_{1 \rightarrow 2}=\exp \left[-\left(E_{2}-q v_{i, j}\right) / k_{B} T\right]
$$

and:

$$
W_{2 \rightarrow 1}=\exp \left[-E_{1} / k_{B} T\right]
$$

where $E_{1}, E_{2}$ are two activation energies, $q$ is an effective charge and $k_{B}$ is the Boltzmann constant. In other terms, each resistor behaves as a generator of a random telegraph signal [21] with voltage dependent transition rates. Therefore, by adopting a mean field-like approach, similar to that described in Ref. [22], for $W_{2 \rightarrow 1} \ll 1$ the average value $\langle p\rangle$ of $p$ can be written as:

$$
\langle p\rangle \approx \frac{1}{1+\frac{\left\langle W_{2 \rightarrow 1}\right\rangle}{\left\langle W_{1 \rightarrow 2}\right\rangle}} \equiv \frac{1}{1+\exp (a-b v)}
$$

where: $a \equiv\left(E_{2}-E_{1}\right) / K_{B} T, b \equiv q / K_{B} T$ and $v \equiv\langle v\rangle$ is the average voltage applied to an elementary resistor. Moreover, it is possible to give

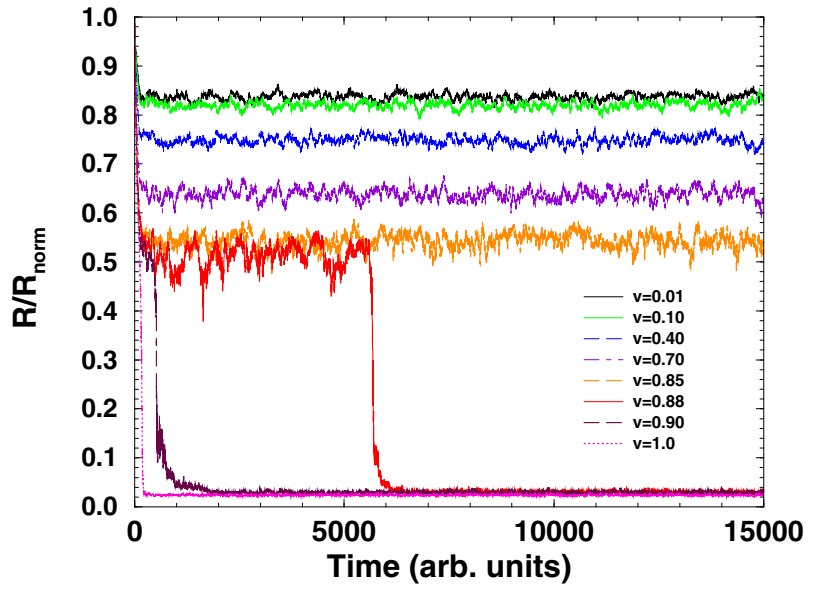

Fig. 1. Typical resistance evolutions calculated for several values of the applied voltage, ranging from $0.01 \mathrm{~V}$ (upper curve) to $1.0 \mathrm{~V}$ (lower curve). The values of the model parameters are given in the text. The resistance is normalized to the perfect network value.

an approximate analytical expression for the relative resistance noise of the network:

$$
\Phi(v) \equiv \frac{\left\langle(\delta r)^{2}\right\rangle}{\langle r\rangle^{2}} \approx \frac{1-\langle p\rangle}{\langle p\rangle} \frac{\left(r_{1}-r_{2}\right)^{2}}{\left[\frac{1-\langle p\rangle}{\langle p\rangle} r_{1}+r_{2}\right]^{2}}
$$

Thus:

$$
\Phi(v) \approx \frac{\left(f_{r}-1\right)^{2} \exp (a-b v)}{\left[f_{r} \exp (a-b v)+1\right]^{2}}
$$

where $f_{r}=r_{1} / r_{2}$. Equation (5) shows that the relative noise of the network depends only on the three parameters: $f_{r}, a, b$ and on the size $N$ (which enters in the average value $v$ ). The evolution of the network is calculated by numerical simulations performed according to an iterative procedure similar to that described in [20] and by using constantvoltage boundary conditions. Here we report results obtained by taking the following values of the model parameters (chosen as reasonable values): $N=75, f_{r}=290, a=2.5$ and $b=210 \mathrm{~V}^{-1}$.

\section{RESULTS}

Figure 1 shows the time evolution of the network resistance $R$ (the time is expressed in iteration steps). Several $R(t)$ signals are reported corresponding to increasing voltage values, ranging from the 


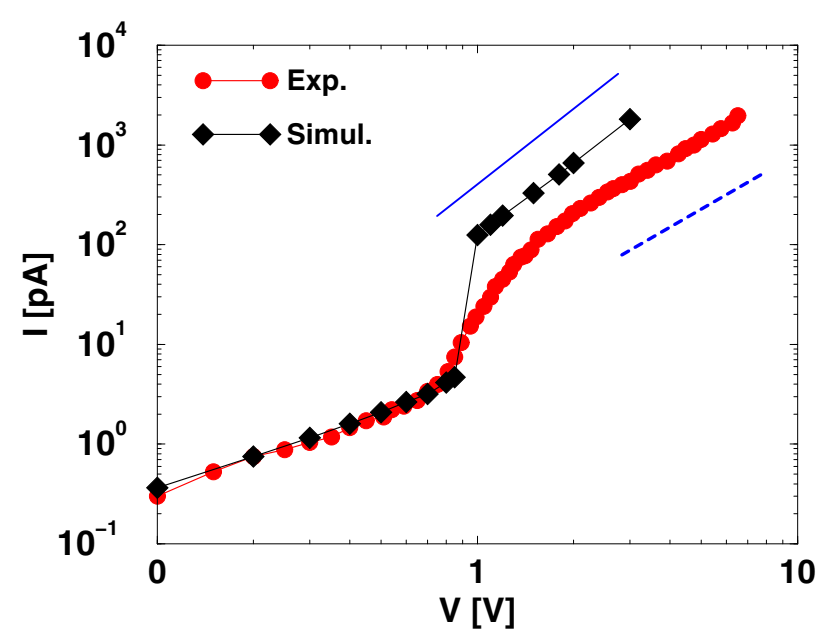

Fig. 2. Red circles: experimental $I-V$ characteristic measured in a tetracene sample (Ref. [14] ). Black diamonds: $I-V$ characteristic obtained from numerical simulations based on the model described in the text. The dashed and the continuous curves refer to the slopes $1.9 \pm 0.1$ and $2.4 \pm 0.1$, respectively

Ohmic regime (upper curve) up to the beginning of the SCLC regime (lower curve). We can see that a rather abrupt transition occurs for voltage values in the range $0.7 \div 1.0 \mathrm{~V}$. In particular, we notice that by approaching the transition region, metastable states of the network exist with relaxation times first increasing with voltage and then drastically decreasing.

Figure 2 displays the calculated $I-V$ characteristic curve. Each $I$ value has been obtained by averaging the $R$ values over the signal $R(t)$ (only the stationary portion of the signal, after the decay of the metastable state, has been considered). For comparison the $I-V$ curve measured in a tetracene sample at $300 \mathrm{~K}$ [14] has been also reported. After the Ohmic regimes, both the curves are characterized by a strong superlinearity in the transition region. The slopes of the SCLC regimes are $1.9 \pm 0.1$ for experimental data (lowest dashed curve) and $2.4 \pm 0.1$ for the simulated one (highest continuous curve).

Figure 3 reports the calculated values of the relative resistance noise of the network as a function of the applied voltage. Again, for comparison we plot also the experimental curve of the relative spectral density of current noise at $20 \mathrm{~Hz}$ measured for the same tetracene sample of Fig. 2 [14]. Overall, Fig. 2 and Fig. 3 show a satisfactory agreement between

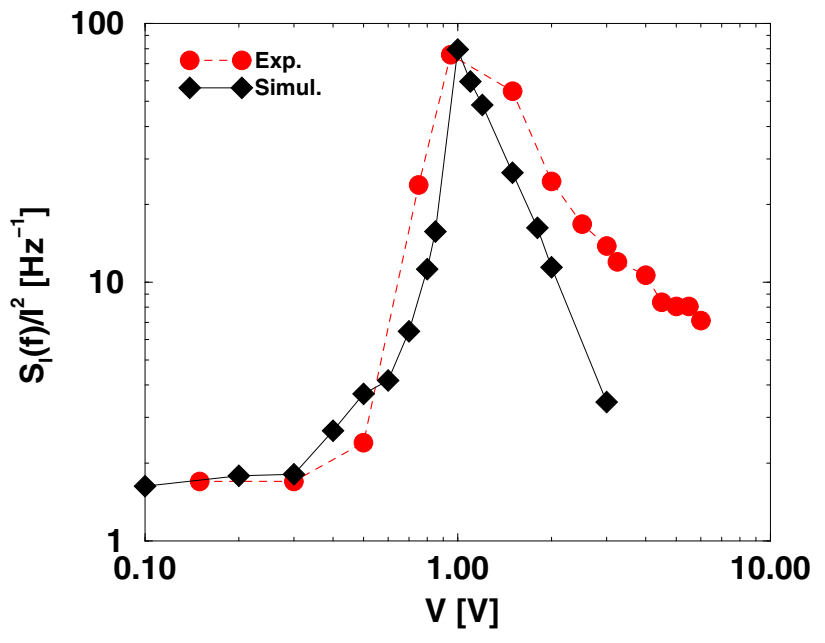

Fig. 3. Red circles: experimental values of $S(f)$ (ref. [14]). Black diamonds: relative current noise $S_{\text {sim }}$ obtained from numerical simulations. The simulated values are normalized by a factor $S_{\text {norm }}=5.26 \times 10^{-5}$ to agree with the measured value of $S_{\max }$.

experiments and the model predictions.

Figure 4 displays the average fraction $\langle p\rangle$ of resistors in the state 2 as a function of the voltage applied to the network. The abrupt change of $\langle p\rangle$ for $V \approx 0.9 \mathrm{~V}$ points out the first order character of the transition [23].

This abroupt variation of the average fraction $\langle p\rangle$ has its counterpart in the sharp peak of the variance of $p$ itself shown in Fig. 5 for the same voltage value. Thus, Fig. 5 highlights the key role played by the fluctuations of the $p$ fraction due to TD processes, as described by Eq. (1) and (2), in the peak of relative noise shown in Fig. 3 and observed in the experiments.

\section{CONCLUSION}

In conclusion, we have considered the $I-V$ characteristic and the sharp peak of the relative spectral density of current noise observed as a function of the applied voltage in polyacene semiconductors [14]. A qualitative explanation of these findings was initially proposed in terms of a continuous percolation between a Ohmic phase and an insulating SCLC phase. Recently [18], we have advanced an alternative interpretation of this behavior, based on a phenomenological model, in terms of trapping and detrapping (TD) processes of the injected car- 


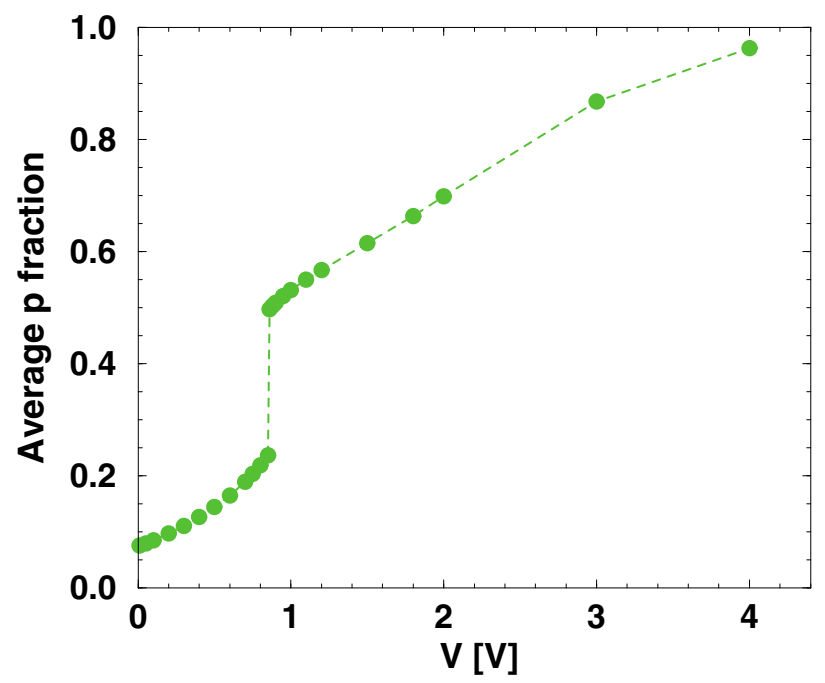

Fig. 4. Average fraction $p$ of $r_{2}$ resistors calculated as a function of the applied voltage. The dashed line is a guide to the eyes.

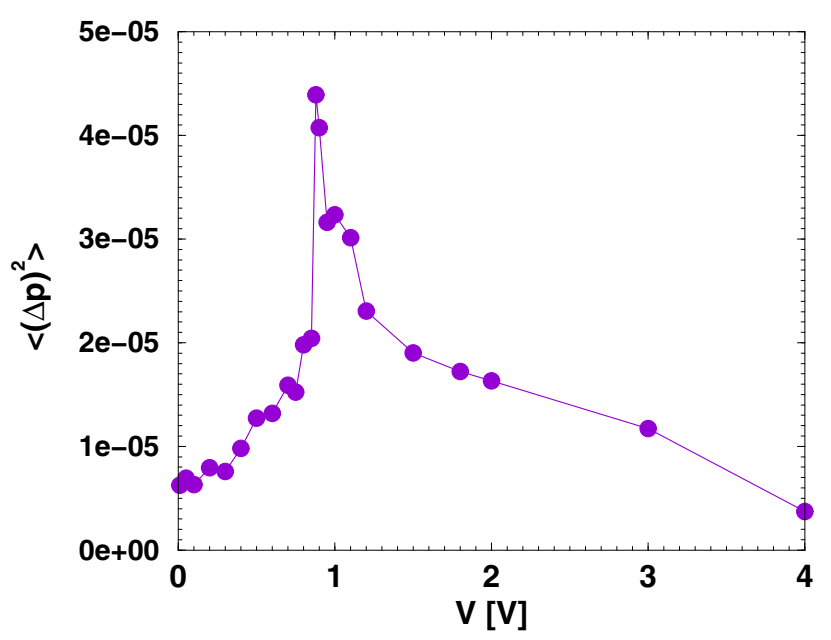

Fig. 5. Variance of the $r_{2}$ resistor fraction calculated as a function of the applied voltage. The dashed line is a guide to the eyes.

riers. To check these two alternative explanations, we have developed a new percolation model. The results of our numerical simulations support the conclusions of the phenomenological model.

\section{ACKNOWLEDGMENT}

This research is partially supported by the European Commission under the Bioelectronic Olfactory Neuron Device (BOND) project within the grant agreement number 228685-2.

\section{REFERENCES}

[1] M. Pope and C. E. Swemberg, Electronic Processes in Organic Crystals and Polymers, Oxford, Oxford University Press (1998).

[2] C.M. Van Vliet, J. Appl. Phys.., 93, 6068 (2003).

[3] D. V. Lang et al., Phys. Rev. Lett., 93, 076601 (2004).

[4] T. Miyadera et al., Appl. Phys. Lett., 93, 033304 (2008).

[5] N. Koch et al., Appl. Surf. Sc., 244, 593 (2005).

[6] Y. S. Yang et al., Appl. Phys. Lett., 80, 1595 (2002).

[7] D. Knipp et al., Appl. Phys. Lett., 82, 3907 (2003).

[8] F. Dinelli et al., Phys. Rev. Lett., 92, 116802 (2004).

[9] C. H. Schwalb et al., Phys. Rev. Lett. , 101, 146801 (2008).

[10] R. W. I. De Boer et al., J. Appl. Phys. , 95, 1196 (2004).

[11] J. H. D. Kang et al., Appl. Phys. Lett., 86, 152115 (2005)

[12] W. Chandra et al., Appl. Phys. Lett., 90, 153505 (2007).

[13] M. Giulianini et al., Appl. Phys. Lett., 94, 083302 (2009) and I. Torres et al., Appl. Phys. Lett., 85, 314 (2004).

[14] A. Carbone et al. Phys. Rev. Lett. 95, 236601 (2005) and A. Carbone Eur. Phys. J. B 50, 77 (2006).

[15] O. D. Jurchescu et al., Appl. Phys. Lett., 92, 132103 (2008).

[16] V. Necliudov et al., J. Appl. Phys., 88, 5395 (2000); S. Martin et al., J. Appl. Phys., 87, 3381 (2000); M. Sampietro at al., J. Appl. Phys. Lett., 78, 3262 (2001).

[17] T. M. G. Kleinpenning Physica B,C 94, 141 (1978) and F. N. Hooge et al. Rep. Prog. Phys. 44, 479 (1981).

[18] A. Carbone et al., Appl. Phys. Lett., 95, 233303 (2009).

[19] D. Stauffer and A. Aharony, Introduction to Percolation Theory, Taylor and Francis (1992).

[20] C. Pennetta Fluctuation and Noise Letters, 2, R29 (2002).

[21] Sh. Kogan, Electronic Noise and Fluctuations in Solids, Cambridge Univ. Press, Cambridge (1996).

[22] C. Pennetta et al., J. Stat. Mech., p02053 (2009).

[23] C. Pennetta et al. , Physica A, 340, 380 (2004). 